\title{
Term weighting scheme effect in sentiment analysis of online movie reviews
}

\begin{abstract}
Sentiment analysis is an evolving field of a study that deals directly with the online expressions posted by the user via the Internet with the main objective to automate the process of mining opinions into valuable information. For online reviews, this analysis deals with the identification of positive and negative reviews to help the consumer and the distributor in the decision-making process. In text analysis tasks, such as text classification and sentiment analysis, the appropriate choice of term weighting schemes will have a huge impact on the effectiveness of the analysis. This paper explores the effect of using term weighting scheme in the sentiment classification of online movie reviews. Specifically, the researchers applied Support Vector Machine (SVM) with linear and non-linear kernels to perform the classification process. The main finding of this study was that LinearSVC when used with TF-IDF improved the classification performance by as much as $87 \%$. Thus, LinearSVC, together with TF-IDF, can serve as an effective technique in the extraction process of online documents.
\end{abstract}

Keyword: Movie reviews; SVM; Sentiment analysis; TF-IDF; Term weighting 